\title{
Expression and DNA methylation changes in human breast epithelial cells after bisphenol A exposure
}

\author{
SANDRA V. FERNANDEZ ${ }^{1}$, YONG HUANG ${ }^{3}$, KARA E. SNIDER $^{1}$, \\ YAN ZHOU $^{2}$, THOMAS J. POGASH ${ }^{1}$ and JOSE RUSSO ${ }^{1}$ \\ ${ }^{1}$ Breast Cancer Research Laboratory; ${ }^{2}$ Department of Biostatistics and Bioinformatics, Fox Chase Cancer Center, \\ Philadelphia, PA 19111; ${ }^{3}$ Center for Biomedical Informatics, University of Chicago, Chicago, IL 60637, USA
}

Received January 30, 2012; Accepted March 12, 2012

DOI: $10.3892 /$ ijo.2012.1444

\begin{abstract}
It has been suggested that xenoestrogens, a group of agents termed endocrine disruptors, may contribute to the development of hormone-dependent cancers, such as breast and endometrial cancers. We previously demonstrated that the xenoestrogen, bisphenol A (BPA), was able to induce the transformation in vitro of human breast epithelial cells. The normal-like human breast epithelial cell line, MCF-10F, formed tubules in collagen (3-D cultures), although after treatment with BPA $\left(10^{-5} \mathrm{M}\right.$ and $\left.10^{-6} \mathrm{M} \mathrm{BPA}\right)$ the cells produced less tubules (73\% and $80 \%$, respectively) and some spherical masses $(27 \%$ and $20 \%$, respectively). In the present study, expression and DNA methylation analyses were performed in these cells after exposure to BPA. These cells showed an increased expression of BRCA1,BRCA2, BARD1, CtIP, RAD51 and BRCC3, all of which are genes involved in DNA repair, as well as the downregulation of PDCD5 and BCL2L11 (BIM), both of which are involved in apoptosis. Furthermore, DNA methylation analysis showed that the BPA exposure induced the hypermethylation of $B C L 2 L 11$, $P A R D 6 G, F O X P 1$ and SFRS11, as well as the hypomethylation of NUP98 and CtIP (RBBP8). Our results indicate that normal human breast epithelial cells exposed to BPA have increased expressions of genes involved in DNA repair in order to overcome the DNA damage induced by this chemical. These results suggest that the breast tissue of women with BRCA1 or BRCA2 mutations could be more susceptible to the effects of BPA.
\end{abstract}

\section{Introduction}

Bisphenol A (BPA) is an environmental contaminant due to the fact that it is a monomer that is polymerized to manufacture polycarbonate plastic and epoxy resins. Polycarbonate plastic is used to make baby and water bottles, dental fillings and seal-

Correspondence to: Sandra V. Fernandez, Breast Cancer Research Laboratory, Fox Chase Cancer Center, 333 Cottman Avenue, Philadelphia, PA 19111, USA

E-mail: sandra.fernandez@fccc.edu

Key words: bisphenol A, xenoestrogens, breast cancer, DNAmethylation, BRCA1, DNA repair ants; epoxy resins are used as coatings on the inside of almost all food and beverage cans $(1,2)$. Thus, BPA leaches into food and beverages through the use of tin cans and polycarbonate plastic containers. The rate of leaching increases when polycarbonate is scratched and discolored (3-5). Decades of continuous release of free BPA into food, beverages and the environment have resulted in a widespread human exposure to this chemical.

BPA is lipophilic and it has been detected in breast adipose tissue samples (6). It has also been detected in human urine at concentrations of $\geq 0.1 \mu \mathrm{g} / 1$ (3-5,7-12). BPA has also been found in maternal plasma $(3.1 \mathrm{ng} / \mathrm{ml})$, fetal plasma $(2.3 \mathrm{ng} / \mathrm{ml} ; \sim 10 \mathrm{nM})$, placental tissues (1-104.9 $\mathrm{ng} / \mathrm{g})$ and amniotic fluid $(8 \mathrm{ng} / \mathrm{ml})$, indicating that there is a significant exposure of pregnant women and their fetuses to BPA $(8,11)$.

In vivo studies have shown that, in rodents, early-life exposure to BPA results in persistent alterations in mammary gland morphogenesis and increased susceptibility to tumorigenesis $(13,14)$. In rats, maternal exposure to BPA during lactation has been shown to decrease time to first tumor latency and increase the number of 7,12-dimethylbenz(a)anthracene (DMBA)-induced mammary tumors in their female offspring (15). However, there is less evidence of the carcinogenic activity of BPA when administered to adult animals. Studies using human breast cancer cells have shown inconsistent data as regards the mitogenic, apoptotic and transcriptional properties of BPA (16-19). This inconsistency is attributed to the lack of the linear dose-dependence of BPA, which often shows a 'U'- or an inverted 'U'-shaped curve (20).

In a previous study, we investigated the effect of BPA on the normal-like human breast epithelial cell line, MCF-10F (21). These cells form tubules in collagen (3-D cultures) resembling the ducts of the normal mammary gland (22). We showed that the treatment of MCF- $10 \mathrm{~F}$ cells with $10^{-5} \mathrm{M}$ or $10^{-6} \mathrm{M}$ of BPA was able to decrease the formation of tubules $(73 \%$ and $80 \%$, respectively) and increase the formation of spherical masses in collagen ( $27 \%$ and $20 \%$, respectively), an indication of cell transformation (21). The objective of the present study was to investigate the expression and DNA methylation changes in MCF-10F cells after BPA exposure.

\section{Materials and methods}

Cells and treatments. MCF-10F is a normal-like human breast epithelial cell line that is estrogen receptor $\alpha(\mathrm{ER} \alpha)$ - and proges- 
terone receptor $(\mathrm{PgR})$-negative. $\mathrm{MCF}-10 \mathrm{~F}$ was maintained in DMEM: F12 medium (1:1 Gibco-BRL, Gaithersburg, MD, USA) supplemented with $5 \%$ horse serum (Gibco), $100 \mathrm{ng} / \mathrm{ml}$ cholera toxin (ICN Biomedicals, Cleveland, OH, USA), $10 \mu \mathrm{g} / \mathrm{ml}$ insulin (Sigma-Aldrich, St. Louis, MO, USA), $0.5 \mu \mathrm{g} / \mathrm{ml}$ hydrocortisone (Sigma-Aldrich), $20 \mathrm{ng} / \mathrm{ml}$ epidermal growth factor (Gibco), $1.05 \mathrm{mM} \mathrm{CaCl}_{2}$ and antibiotics (penicillin, $100 \mathrm{U} / \mathrm{ml}$; streptomycin, $100 \mu \mathrm{g} / \mathrm{ml}$; amphotericin, $0.25 \mu \mathrm{g} / \mathrm{ml}$; Sigma-Aldrich). BPA (from Sigma-Aldrich) was dissolved in dimethyl sulfoxide (DMSO) and the cells were treated with $10^{-5} \mathrm{M}$ or $10^{-6} \mathrm{M}$ BPA continuously for 2 weeks as described previously (21). As controls, MCF-10F cells were not treated and maintained in the regular medium or treated with $0.284 \%$ DMSO (21). After BPA treatment, the cells were expanded and RNA or DNA were isolated for expression and DNA methylation studies, respectively.

Expression arrays. RNA was isolated from the cells using RiboPure $^{\mathrm{TM}}$ kit (Life Technologies, Carlsbad, CA, USA). The genome-wide gene expressions were performed using the Human Genome U133 Plus 2.0 arrays (Affymetrix, Santa Clara, CA, USA). The arrays were performed in duplicate for the MCF-10F cells treated with $10^{-6} \mathrm{M}$ and $10^{-5} \mathrm{M}$ BPA and for the control (cells without BPA treatment). After hybridization, the chips were scanned using GeneChip Scanner 3000.

Methylated DNA immunoprecipitation-on-chip (MeDIP-onchip). MeDIP-on chip consists of an immunocapturing approach for enriching methylated DNA in combination with detection by DNA microarray. DNA was isolated from cells treated with $10^{-5} \mathrm{M}, 10^{-6} \mathrm{M} \mathrm{BPA}$, and the control MCF-10F cells (without BPA treatment) using the DNeasy Blood and Tissue kit (Qiagen, Valencia, CA, USA). The DNA was fragmented (150-500 bp) by sonication and methylated DNA was immunoprecipitated with a monoclonal antibody against 5-methylcytidine (Eurogentec, San Diego, CA, USA) (23). Methylated fragments were amplified using the GenomePlex Whole Genome Amplification kit (WGA, Sigma-Aldrich) (23). Double-stranded DNA was treated by a combination of UDG and APE1 that specifically recognizes the dUTP residues and breaks the DNA into fragments. Targets were then labeled with Affymetrix labeling reagent and terminal deoxynucleotidyl transferase (TdT) for $1 \mathrm{~h}$. The mixtures were hybridized to the Human promoter 1.0R Array (Affymetrix) that comprises more than 4.6 million probes tiled to interrogate more than 25,500 genes; it interrogates $7.5 \mathrm{~kb}$ upstream and $2.5 \mathrm{~kb}$ downstream of transcription start sites. The methylation arrays were performed in duplicate for the cells treated with $10^{-6} \mathrm{M} \mathrm{BPA}$ and in triplicate for the cells treated with $10^{-5} \mathrm{M}$ BPA.

Data analyses. The mRNA expression arrays were analyzed using the Bioconductor 'limma' package. Briefly, the raw data were normalized using the 'rma' method (24). Differentially expressed genes were identified using empirical Bayes methods implemented into the limma package (25). The criteria for significance are determined by a fold change of $>2$ and a Benjamini-Hochberg false discover rate of $<5 \%$. The functional significance of up- or downregulated genes in the BPA-treated cells were analyzed using Ingenuity Pathways Analysis software (IPA) version 5.0 (Ingenuity Systems, Redwood City,
Table I. Expression values of certain genes in MCF-10F cells treated with $10^{-6} \mathrm{M}$ BPA.

\begin{tabular}{|c|c|c|}
\hline Gene symbol & GenBank & Fold change \\
\hline ALDH1A3 & NM_000693 & 20.82 \\
\hline AURKA & NM_003600 & 23.43 \\
\hline AURKB & AB011446 & 4.99 \\
\hline BARD1 & NM_000465 & 4.50 \\
\hline BCL11A & AF080216 & 0.13 \\
\hline BCL2L11 (BIM) & AA629050 & 0.12 \\
\hline BCL2L13 & AA 156605 & 0.42 \\
\hline BOLA3 & AI380704 & 3.58 \\
\hline BRCA1 & NM_007295 & 24.25 \\
\hline BRCA2 & NM_000059 & 6.82 \\
\hline BRCC3 & X64643 & 5.43 \\
\hline $\mathrm{CD} 24$ & X69397 & 9.25 \\
\hline CD44 & AL552534 & 0.18 \\
\hline CEACAM1 & X16354 & 317.37 \\
\hline CtIP (RBBP8) & NM_002894 & 2.06 \\
\hline ERBB3 & NM_001982 & 6.73 \\
\hline FN1 & AK026737 & 11.96 \\
\hline HDAC5 & NM_005474 & 0.02 \\
\hline HDAC8 & AF212246 & 6.06 \\
\hline HDAC9 & NM_014707 & 0.11 \\
\hline ID2 & AI819238 & 17.03 \\
\hline IL18R1 & NM_003855 & 0.04 \\
\hline JAG1 & U73936 & 0.35 \\
\hline JAG2 & Y14330 & 0.26 \\
\hline LOX & NM_002317 & 0.29 \\
\hline MTSS1 & NM_014751 & 0.02 \\
\hline MUC1 & AF348143 & 3.81 \\
\hline MUC16 & NM_024690 & 124.50 \\
\hline MUC20 & AW084511 & 21.26 \\
\hline MYCBP & D50692 & 15.78 \\
\hline PARD6G & AI817448 & 0.06 \\
\hline PDCD5 & AI817145 & 0.04 \\
\hline RAD51 & NM_002875 & 36.25 \\
\hline RARRES1 (TIG1) & NM_002888 & 1016.92 \\
\hline RARRES3 (TIG3) & NM_004585 & 3.97 \\
\hline SFRP1 & AF017987 & 0.34 \\
\hline SLIT2 & AF055585 & 0.22 \\
\hline SMAD5 & AF010601 & 0.19 \\
\hline TSPAN5 & AA059445 & 0.02 \\
\hline TWIST1 & X99268 & 0.09 \\
\hline VIM & AI922599 & 0.02 \\
\hline
\end{tabular}

Genes that are at least 2-fold up- or downregulated (fold change) are indicated.

CA, USA). The differentially expressed genes were uploaded into IPA to identify significantly enriched canonical pathways. The significance of a canonical pathway is controlled by the p-value calculated using the right-tailed Fisher's exact test for $2 \times 2$ contingency tables.

The MeDIP-on chip data were analyzed using CisGenome Software, an integrated tool package for tiling array and ChIP-seq analysis (26). Two-sample analyses were performed using BPA-treated cells versus MCF-10F control cells (without BPA treatment) precipitated using the antibody against 5-methyl- 
A

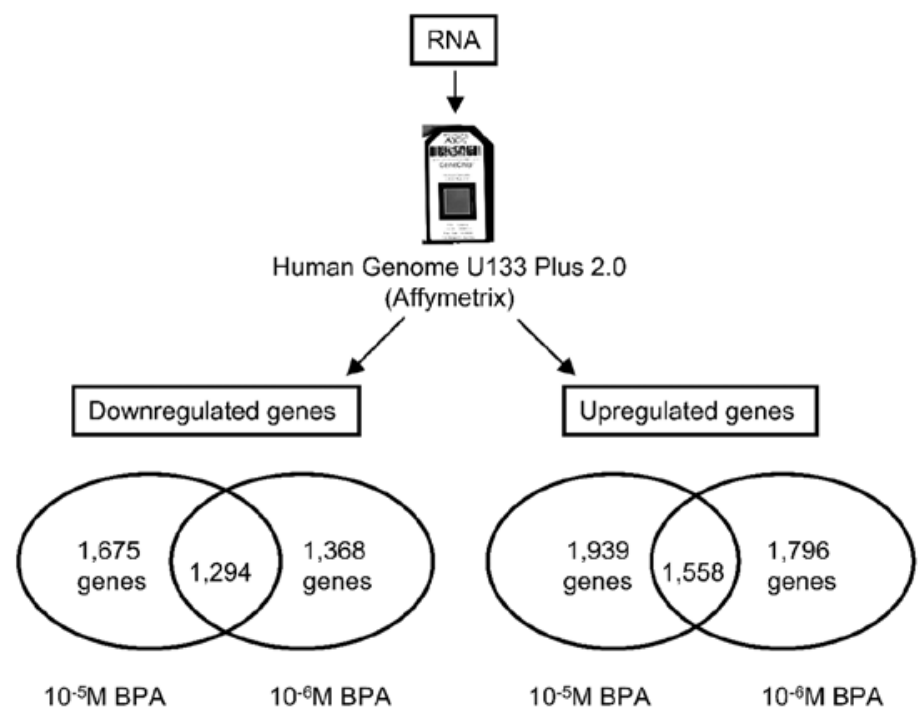

B

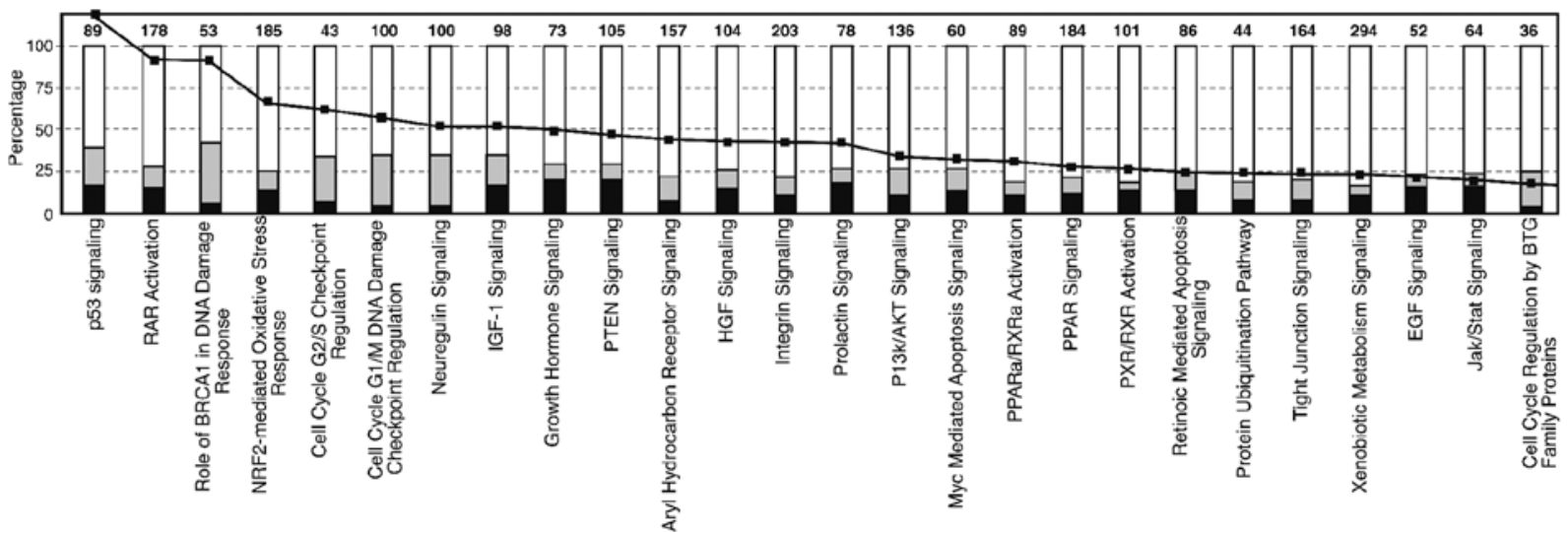

Figure 1. Expression studies of the MCF-10F cells exposed to BPA. (A) RNA was isolated from the cells treated with $10^{-5} \mathrm{M}$ and $10^{-6} \mathrm{M}$ BPA and expression analyses were performed using the Human Genome U133 Plus 2.0 arrays (Affymetrix). (B) Canonical pathways enriched with deregulated genes in MCF-10F cells exposed to $10^{-6} \mathrm{M}$ BPA. Black, number of downregulated genes; gray, number of upregulated genes; black line, $\log$ (p-value).

cytidine. Data were quantile normalized before the comparison. The ChIP-chip peak calling was detected with the Model-based Analysis of Tiling-array (MAT) algorithm (27) integrated in CisGenome. MAT was run with the following parameters to capture regions of increased signal intensity: a bandwidth of 300 , a maximum gap of 300 , a max run of insignificant probes within a region of 5, a minimum region length of 100 , a minimal number of significant probes within a region of 5 and window p-value cut-off of 0.0001 . Hyper- or hypomethylated regions were determined by the signals of BPA-treated cells which were significantly higher or lower than those of the MCF-10F control cells. The MAT library and mapping files based on the March 2006 Human Genome Assembly (HG18) were used to link ChIP Hits to RefSeq genes. Briefly, the files containing transcription start site (TSS) and end-point were linked to the RefSeq table with accession numbers. Chromosomal positions were then used to associate ChIP hits with RefSeq gene IDs. Specifically, hits falling within a window of -0.5 to $+2.5 \mathrm{~kb}$ of a given RefSeq TSS were annotated as being associated with that gene. To illustrate the impact of methylation on gene expression, the hyper- or hypomethylated genes were compared to the down- or upregulated genes determined by the expression array. The raw data were submitted to the NCBI GEO data base with accession numbers GSE26884 and GSE27865 for the expression and methylation arrays, respectively.

\section{Results}

Expression studies of the human breast epithelial cells after $B P A$ exposure. We characterized the cells exposed to $10^{-5} \mathrm{M}$ or $10^{-6} \mathrm{M}$ BPA by expression studies using microarrays. MCF-10F cells exposed to $10^{-5} \mathrm{M}$ or $10^{-6} \mathrm{M}$ BPA showed 3,614 and 3,164 deregulated genes (up- or downregulated compared to the MCF-10F control), respectively (Fig. 1A). We found a total of 1,675 genes downregulated in the cells exposed to $10^{-5} \mathrm{MBPA}$ and 1,368 genes downregulated in the cells exposed to $10^{-6} \mathrm{M}$ BPA; from these genes 1,294 genes were found to be downregulated with both BPA concentrations (Fig. 1A). A total of 1,939 genes were upregulated in the cells treated with $10^{-5} \mathrm{M}$ BPA and 1,796 genes were upregulated in the cells exposed to $10^{-6} \mathrm{M} \mathrm{BPA}$; from these genes, 1,558 genes were upregulated in the cells treated with both concentrations (Fig. 1A).

The functional enrichment of up- or downregulated genes in the BPA-exposed cells was analyzed using IPA software. Gene 
A

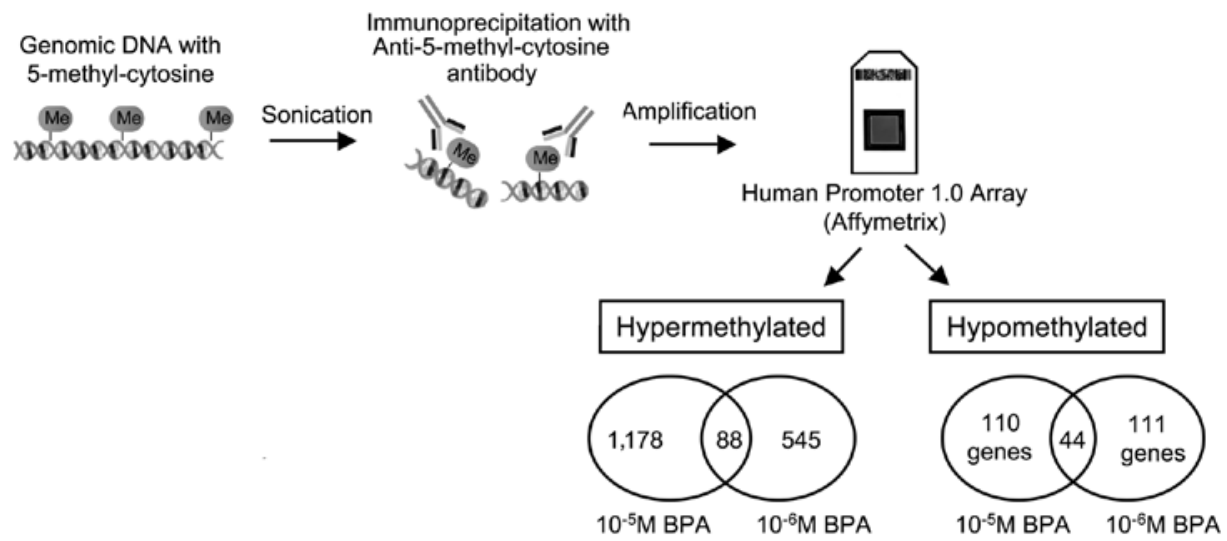

B

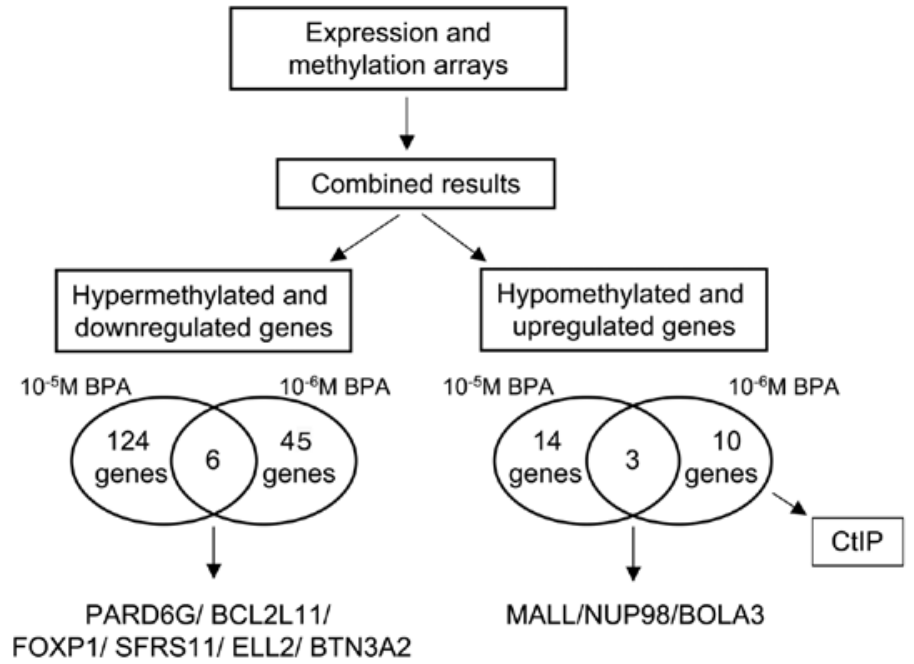

Figure 2. DNA methylation studies in MCF-10F cells exposed to BPA. (A) DNA was isolated from the cells and fragmented by sonication. Methylated DNA was isolated using an antibody against 5-methylcytidine and amplified, followed by hybridization using a promoter microarray to identify regions with altered methylation in the promoters. The Human Promoter 1.0R Arrays were used and hyper- and hypomethylated promoters were identified. Hypermethylated targets were sequences with significantly increased signals in the cells exposed to BPA relative to the control without treatment immunoprecipitated using the antibody against 5-methylcytidine. (B) Combined results from the expression and DNA methylation arrays: the list of genes that were found hypermethylated by MeDIP-on-chip and the list of genes found downregulated by expression arrays were compared. Left, the number of genes hypermethylated and downregulated is indicated for the cells exposed to $10^{-5} \mathrm{M}$ and $10^{-6} \mathrm{M}$ BPA. The same was done for genes hypomethylated and upregulated. Right, the number of genes hypomethylated and upregulated is indicated for the cells treated with $10^{-5} \mathrm{M}$ and $10^{-6} \mathrm{M}$ BPA.

networks and canonical pathways representing key genes were identified. The canonical pathways more affected in the cells exposed to BPA were the DNA damage response, p53 signaling (activated by genotoxic or non-genotoxic stress), the retinoic acid receptor activation, and the neuregulin signaling pathways (Fig. 1B). Cells treated with $10^{-5} \mathrm{M}$ or $10^{-6} \mathrm{M}$ BPA showed decreased expressions of PDCD5 and BCL2L11 (also known as $B I M$ ), both of which are involved in apoptosis, and an increased expression of BRCAl, BARDI, CtIP (also known as RBBP8), $R A D 51$ and $B R C C 3$, all of which are involved in DNA repair.

Table I shows genes which were at least 2-fold up- or downregulated in the cells after exposure to $10^{-6} \mathrm{M} \mathrm{BPA}$. A number of genes involved in DNA damage response were upregulated, such as BRCA1 (24.25-fold induction), BRCA2 (6.82-fold), BRCC3 (5.43-fold), BARDI (4.5-fold), CtIP (2.06-fold) and RAD51 (36.25-fold). Other genes downregulated after exposure to $10^{-6} \mathrm{M}$ BPA were: $J A G 1$ (0.35-fold induction), JAG2 (0.26-fold), SMAD5 (0.19-fold), TWIST1 (0.09-fold), VIM (0.02-fold), TSPAN5 (0.02-fold), CD44 (0.18-fold) and HDAC5 (0.02-fold)
(Table I). Exposure to $10^{-6} \mathrm{M}$ BPA induced the overexpression of RARRES1 (1016.92-fold) and RARRES3 (3.97-fold), both of which involved in the retinoic acid receptor pathway (Table I). Some upregulated genes were CEACAM1 (317.37-fold), ALDH1A3 (20.82-fold), AURKA (23.43-fold), ID2 (17.03-fold), FN1 (11.96-fold induction) and CD24 (9.25-fold) (Table I).

DNA methylation studies of MCF-10F cells after BPA exposure. We studied DNA methylation changes in the MCF-10F human breast epithelial cells after exposure to BPA using MeDIP-on-chip (23). Gene regulatory regions that were hypoor hypermethylated were identified. Hypermethylated targets were sequences with significantly increased signals in the cells exposed to BPA relative to the control cells (MCF-10F growth without treatment). The DNA from the BPA-treated cells and the control cells was immunoprecipitated with the antibody against 5-methyl-cytosine. The cells treated with $10^{-5} \mathrm{M}$ BPA showed 1,178 genes hypermethylated and those treated with $10^{-6} \mathrm{M}$ BPA showed 545 hypermethylated genes; from these 
Table II. Downregulated and hypermethylated genes in MCF-10F cells treated with $10^{-5} \mathrm{M}$ BPA.

\begin{tabular}{|c|c|c|c|c|c|c|c|}
\hline $\begin{array}{l}\text { Gene } \\
\text { symbol }\end{array}$ & $\begin{array}{c}\text { Fold } \\
\text { change }\end{array}$ & $\begin{array}{c}\text { Gene } \\
\text { symbol }\end{array}$ & $\begin{array}{c}\text { Fold } \\
\text { change }\end{array}$ & $\begin{array}{c}\text { Gene } \\
\text { symbol }\end{array}$ & $\begin{array}{c}\text { Fold } \\
\text { change }\end{array}$ & $\begin{array}{c}\text { Gene } \\
\text { symbol }\end{array}$ & $\begin{array}{c}\text { Fold } \\
\text { change }\end{array}$ \\
\hline SGSH & 0.50 & FADS3 & 0.42 & PURA & 0.34 & ZNF197 & 0.22 \\
\hline OGFRL1 & 0.50 & ABCA12 & 0.42 & RBMS3 & 0.34 & MARK1 & 0.22 \\
\hline IL13RA1 & 0.49 & AFF4 & 0.42 & STK16 & 0.33 & TOMM20 & 0.22 \\
\hline ANKDD1A & 0.49 & MLL & 0.41 & PEX11A & 0.32 & PARD6G & 0.21 \\
\hline UBE2Z & 0.49 & WWOX & 0.41 & SENP6 & 0.32 & C1orf21 & 0.21 \\
\hline PRKAG2 & 0.49 & SLC7A8 & 0.40 & KIAA0182 & 0.32 & BCL2L11 (BIM) & 0.20 \\
\hline ZNF488 & 0.48 & MCEE & 0.40 & PARD3 & 0.31 & PRKCH & 0.20 \\
\hline ZC3H11A & 0.48 & HMHA1 & 0.40 & RNF135 & 0.31 & C7orf31 & 0.19 \\
\hline KCNAB2 & 0.48 & RIOK3 & 0.39 & STAT5B & 0.31 & TMEM91 & 0.19 \\
\hline RHOQ & 0.48 & ELL2 & 0.39 & ATF6 & 0.30 & MGST1 & 0.18 \\
\hline DFFB & 0.47 & C7orf38 & 0.39 & ADARB1 & 0.30 & ANKRD28 & 0.18 \\
\hline TBC1D8B & 0.47 & METTL9 & 0.39 & JAK1 & 0.29 & DHRS3 & 0.18 \\
\hline DGAT2 & 0.47 & FBXO9 & 0.38 & $\mathrm{CP}$ & 0.29 & CCDC11 & 0.16 \\
\hline ZNF219 & 0.47 & EFHC1 & 0.38 & TMEM67 & 0.29 & TMEM37 & 0.15 \\
\hline LRCH3 & 0.47 & CD99L2 & 0.38 & FARP1 & 0.28 & HSD11B1 & 0.15 \\
\hline SH3BP2 & 0.47 & CAPN1 & 0.38 & FAM119B & 0.28 & IL18R1 & 0.13 \\
\hline RUFY1 & 0.47 & SFRS11 & 0.38 & VPS41 & 0.28 & LETMD1 & 0.12 \\
\hline UBE2Q1 & 0.46 & ACVR2A & 0.38 & TMEM80 & 0.28 & FAM19A2 & 0.11 \\
\hline SSR2 & 0.46 & ATP9A & 0.38 & TRIM69 & 0.27 & BBOX1 & 0.10 \\
\hline CIR & 0.46 & DUSP1 & 0.38 & GJA3 & 0.27 & EBF1 & 0.10 \\
\hline GRAMD2 & 0.46 & PPIL6 & 0.38 & IFNGR1 & 0.27 & GBP2 & 0.08 \\
\hline DNAJC16 & 0.46 & MAP2K5 & 0.37 & GPR177 & 0.24 & SULT1E1 & 0.07 \\
\hline HHLA3 & 0.45 & RAB13 & 0.37 & BTN3A2 & 0.24 & TSLP & 0.07 \\
\hline SMYD3 & 0.45 & PKIA & 0.37 & PIK3R1 & 0.24 & PLEKHA6 & 0.07 \\
\hline C5orf 25 & 0.45 & SFXN1 & 0.37 & ZBTB20 & 0.24 & KLHDC8B & 0.07 \\
\hline TMLHE & 0.45 & FGFR1OP2 & 0.37 & TPPP & 0.23 & PDZK1IP1 & 0.06 \\
\hline KIAA1244 & 0.44 & MSX2 & 0.37 & PIR & 0.23 & $\mathrm{DCN}$ & 0.05 \\
\hline ITSN2 & 0.44 & LYPLAL1 & 0.36 & DUSP16 & 0.22 & SORL1 & 0.04 \\
\hline MAPT & 0.44 & KLRK1 & 0.36 & PRKAG2 & 0.22 & ZBTB 10 & 0.03 \\
\hline RNPC3 & 0.44 & GNPTAB & 0.35 & DSC3 & 0.22 & BCL6 & 0.03 \\
\hline FOXP1 & 0.43 & ADRBK2 & 0.34 & ITGA4 & 0.22 & GALNTL2 & 0.003 \\
\hline
\end{tabular}

Hypermethylated genes and their expression values are indicated (fold change).

genes, 88 genes were hypermethylated at both concentrations (Fig. 2A). Hypomethylated targets were sequences that were significantly increased in the control relative to the cells treated with BPA. Using these criteria, we identified 110 genes that were hypomethylated in the cells treated with $10^{-5} \mathrm{M}$ BPA and 111 hypomethylated genes in the cells treated with $10^{-6} \mathrm{M}$ BPA; from these genes, 44 genes were hypomethylated at both concentrations (Fig. 2A).

As hypermethylation is related to gene downregulation and hypomethylation is related to increased gene expression (28), the expression and DNA methylation data were superimposed. The downregulated and hypermethylated genes by BPA exposure were identified (Fig. 2B). In the cells treated with $10^{-5} \mathrm{M}$ BPA, 124 genes were found to be hypermethylated and downregulated (Fig. 2B). In the cells treated with $10^{-6} \mathrm{M}$ BPA, 45 genes were found hypermethylated and downregulated (Fig. 2B). As indicated in Fig. 2B, 6 genes were found to be hypermethylated and downregulated in the cells treated with both $10^{-5} \mathrm{M}$ or $10^{-6} \mathrm{M}$ BPA: PARD6G, BCL2L11 (or BIM), FOXP1, SFRS11, ELL2 and BTN3A2.
In Table II, the 124 genes which were downregulated and hypermethylated in the cells after exposure to $10^{-5} \mathrm{M}$ BPA are indicated; some of these genes were PARD6G (0.21-fold induction), BCL2L11 (0.2-fold), FOXP1 (0.43-fold), SFRS11 (0.38-fold), ELL2 (0.39-fold) and BTN3A2 (0.24-fold). Other genes downregulated and hypermethylated were STAT5B (0.31-fold induction), WWOX (0.41-fold induction) and SULT1E1 (0.07-fold induction). Table III shows the 45 genes downregulated and hypermethylated in the cells after being exposed to $10^{-6} \mathrm{M}$ BPA. Some of these genes were PARD6G (0.16-fold induction), BCL2L11 (0.18-fold), FOXP1 (0.38-fold), SFRS11 (0.22-fold), ELL2 (0.31-fold) and BTN3A2 (0.28-fold). Other genes downregulated and hypermethylated were $R H O U$ (0.02-fold induction), TWIST1 (0.11-fold induction), and SFRP1 (0.39-fold induction).

Similarly, hypomethylated and upregulated genes by BPA were identified (Fig. 2B). In the cells exposed to $10^{-5} \mathrm{M}$ BPA, 14 genes were found to be hypomethylated and upregulated; in the cells treated with $10^{-6} \mathrm{M}$ BPA, 10 genes were found to be hypomethylated and upregulated (Fig. 2B). From these genes, 
Table III. Downregulated and hypermethylated genes in MCF-10F cells treated with $10^{-6} \mathrm{M}$ BPA.

\begin{tabular}{|c|c|c|c|c|c|c|c|}
\hline $\begin{array}{l}\text { Gene } \\
\text { symbol }\end{array}$ & $\begin{array}{c}\text { Fold } \\
\text { change }\end{array}$ & $\begin{array}{c}\text { Gene } \\
\text { symbol }\end{array}$ & $\begin{array}{c}\text { Fold } \\
\text { change }\end{array}$ & $\begin{array}{c}\text { Gene } \\
\text { symbol }\end{array}$ & $\begin{array}{c}\text { Fold } \\
\text { change }\end{array}$ & $\begin{array}{c}\text { Gene } \\
\text { symbol }\end{array}$ & $\begin{array}{c}\text { Fold } \\
\text { change }\end{array}$ \\
\hline NIPA1 & 0.50 & SYNE1 & 0.38 & UACA & 0.24 & MRPL39 & 0.14 \\
\hline EDA & 0.49 & NPL & 0.37 & RAB4A & 0.24 & KLF9 & 0.13 \\
\hline RERE & 0.48 & C17orf69 & 0.36 & SFRS11 & 0.22 & TWIST1 & 0.11 \\
\hline SLC25A28 & 0.47 & TPCN2 & 0.35 & KIAA0564 & 0.21 & RNF128 & 0.10 \\
\hline FAM19A1 & 0.45 & TOP1MT & 0.35 & BCL2L11 (BIM) & 0.18 & DST & 0.10 \\
\hline MGEA5 & 0.44 & TMEM47 & 0.33 & KLHL13 & 0.18 & TOX & 0.07 \\
\hline TLR3 & 0.44 & ELL2 & 0.31 & IGF1R & 0.17 & TTC7B & 0.04 \\
\hline $\mathrm{ZHX} 2$ & 0.41 & PCDHB14 & 0.29 & RPL37 & 0.16 & NXN & 0.04 \\
\hline OSBPL6 & 0.40 & BTN3A2 & 0.28 & PARD6G & 0.16 & RHOU (WRCH1) & 0.02 \\
\hline NUDT2 & 0.40 & TARSL2 & 0.27 & GPC4 & 0.15 & FHL1 & 0.01 \\
\hline SFRP1 & 0.39 & LONRF1 & 0.27 & GAB2 & 0.15 & SOD2 & 0.01 \\
\hline FOXP1 & 0.38 & & & & & & \\
\hline
\end{tabular}

Hypermethylated genes and their expression values (fold change) are indicated.

Table IV. Hypomethylated and upregulated genes in MCF-10F cells treated BPA.

\begin{tabular}{|c|c|c|}
\hline Gene symbol & Gene title & Fold change \\
\hline \multicolumn{3}{|c|}{ Cells treated with $10^{-5} \mathrm{M}$ BPA } \\
\hline MALL & Mal, T cell differentiation protein-like & 67.65 \\
\hline NUP98 & Nucleoporin 98 kDa & 9.65 \\
\hline ARHGAP11A & Rho GTPase activating protein $11 \mathrm{~A}$ & 7.67 \\
\hline BOLA3 & BolA homolog 3 (E. coli) & 5.43 \\
\hline $\mathrm{CA} 2$ & Carbonic anhydrase II & 5.10 \\
\hline GPR172A & $\mathrm{G}$ protein-coupled receptor $172 \mathrm{~A}$ & 4.20 \\
\hline CCDC80 & Coiled-coil domain containing 80 & 3.76 \\
\hline BID & $\mathrm{BH} 3$ interacting domain death agonist & 3.53 \\
\hline NT5E & 5'-Nucleotidase, ecto (CD73) & 3.23 \\
\hline SHC4 & SHC (Src homology 2 domain containing) family, member 4 & 2.73 \\
\hline C12orf30 & Chromosome 12 open reading frame 30 & 2.62 \\
\hline CCDC90A & Coiled-coil domain containing 90A & 2.31 \\
\hline FAM86A & Family with sequence similarity 86 , member A & 2.31 \\
\hline SYNCRIP & Synaptotagmin binding, cytoplasmic RNA interacting protein & 2.25 \\
\hline \multicolumn{3}{|c|}{ Cells treated with $10^{-6} \mathrm{M}$ BPA } \\
\hline MALL & Mal, T cell differentiation protein-like & 72.00 \\
\hline RPL27A & Ribosomal protein L27a & 13.93 \\
\hline GDA & Guanine deaminase & 7.46 \\
\hline NUP98 & Nucleoporin $98 \mathrm{kDa}$ & 5.39 \\
\hline TIAM1 & T cell lymphoma invasion and metastasis 1 & 4.44 \\
\hline BCMO1 & $\beta$-carotene $15,15^{\prime}$-monooxygenase 1 & 4.35 \\
\hline BOLA3 & BolA homolog 3 (E. coli) & 3.10 \\
\hline DDX52 & DEAD (Asp-Glu-Ala-Asp) box polypeptide 52 & 3.01 \\
\hline CtIP (RBBP8) & Retinoblastoma binding protein 8 & 2.06 \\
\hline PPME1 & Protein phosphatase methylesterase 1 & 2.04 \\
\hline
\end{tabular}

Hypomethylated genes and their expression values (fold change) are indicated.

3 were hypomethylated and upregulated in both cells: $M A L L$, NUP98 and BOLA3 (Fig. 2B and Table IV). In the cells treated with $10^{-5} \mathrm{M}$ BPA, MLL, NUP98 and BOLA3 showed a 67.65-, 9.65- and 5.43-fold induction, respectively (Table IV). In the cells treated with $10^{-6} \mathrm{M}$ BPA, MLL, NUP98 and BOLA3 showed a 72-, 5.39- and 3.10-fold induction, respectively (Table III); these cells also showed an upregulation and hypomethylation of CtIP (2.06-fold induction). 


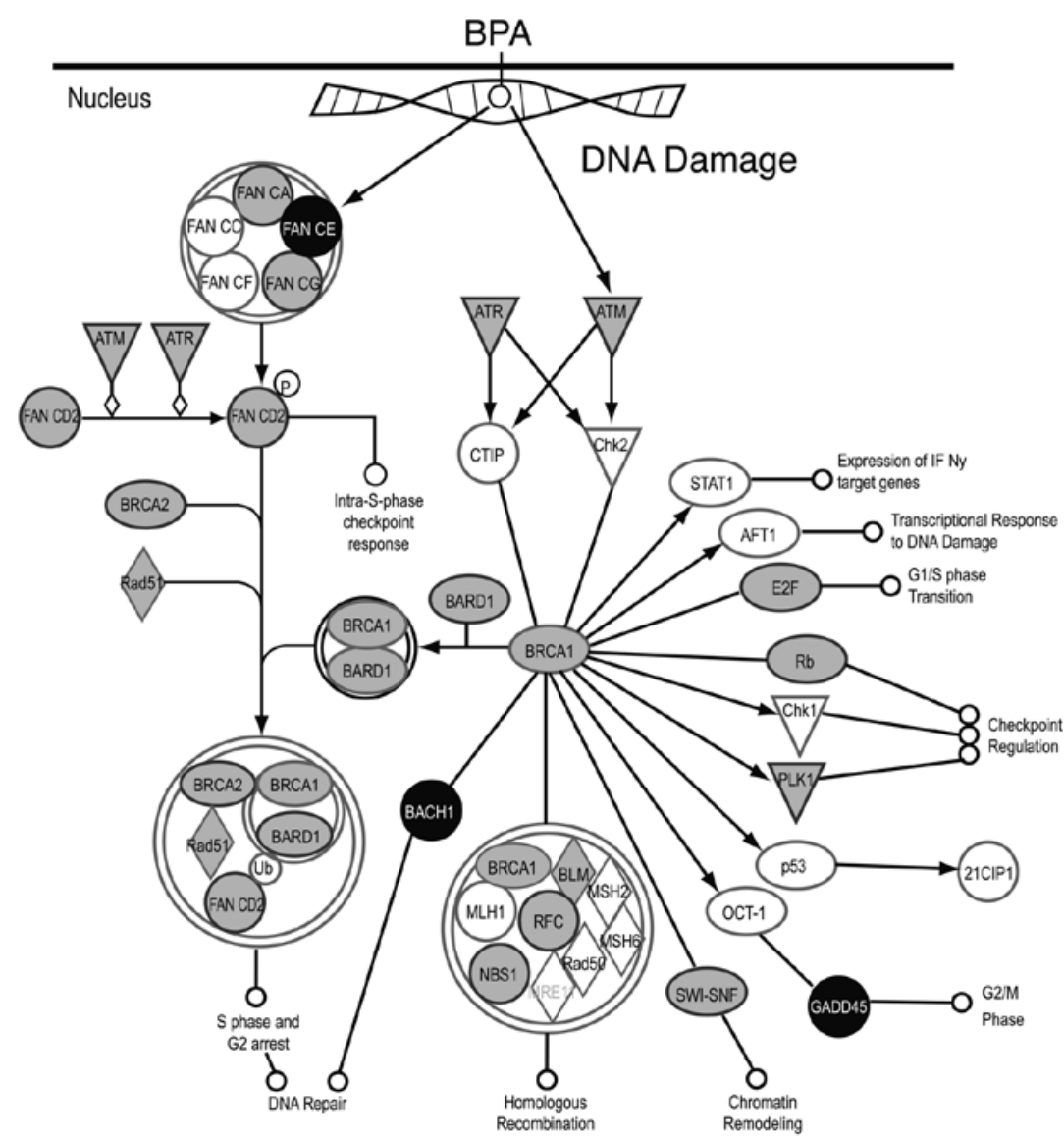

Figure 3. DNA repair genes were induced in the normal breast epithelial cells after being exposed to BPA. The MCF-10F cells showed increased expression of BRCA1, BARD1, BRCA2 and RAD51 after being exposed to $10^{-6} \mathrm{M}$ BPA. Upregulated genes are shown in gray. Downregulated genes are shown in black.

\section{Discussion}

The normal-like human breast epithelial cell line, MCF-10F, showed increased expressions of genes involved in DNA repair (BRCA1, BARDI, CtIP, RAD51 and BRCC3) and decreased expressions of genes involved in apoptosis (PDCD5 and $B C L 2 L 11)$ after being exposed to BPA for 2 weeks. These cells also showed hypermethylation of different genes such as BCL2L11, PARD6G, FOXP1, SFRS11, and hypomethylation of CtIP (or RBBP8) and NUP98.

Epigenetic changes derived from exposure to endocrine disruptors have been described in several tissues and organisms $(29,30)$ although, this is the first demonstration that BPA induces DNA methylation changes in genes related to apoptosis and DNA repair in human breast epithelial cells. BCL2L11 (or $B I M)$ has the ability to trigger apoptosis in various cells, such as epithelial and neuronal cells (31); the fact that this gene was hypermethylated after BPA treatment suggests that apoptosis was inhibited in the cells after exposure to BPA. CtIP was hypomethylated and upregulated after BPA treatment; CtIP is involved in double-strand break (DSB) repair and plays a role in DNA-damage-induced cell cycle checkpoint control at the G2/M transition (32). MCF-10F cells treated with BPA also showed changes in the DNA methylation pattern of partitioning defective 6 homolog $\gamma(P A R D 6 G)$ which is an adapter-protein involved in asymmetrical cell division and cell polarization processes.
It has been suggested that BPA is a weak carcinogen (33). Evidence of the estrogenic effects of BPA has been reported in a number of studies showing that it activates ERs $\alpha$ and $\beta(34,35)$, although the affinity of BPA is at least 10,000-fold less than estrogen for both receptors (36). It has been proposed that BPA, similar to certain estrogen metabolites, can react with DNA to cause mutations that can lead to cancer initiation (33,37-41). One mechanism by which estrogen and BPA initiates breast cancer is by generating adducts that can produce a variety of DNA modifications that, if not countered by DNA repair, can lead to cell transformation. It has been shown that BPA is able to form DNA adducts in vitro and in vivo (42-45); BPA can be converted to bisphenol O-quinone (46) and, the BPA semiquinone and/or quinone intermediates may be the ultimate DNA binding metabolites. In the present study, the ER $\alpha$-negative breast epithelial cell line, MCF-10F, was used indicating that in these cells other mechanisms independent of the ER were responsible for the biological effect of BPA.

In addition to DNA adduct formation, oxidative stress could be another reason for the alterations produced by BPA in the DNA (47). Oxidative DNA lesions include the oxidation of nucleotidic bases, modifications to the sugar moiety of DNA which may result in base-loss abasic (apurinic/apyrimidinic) sites and/or strand breakage (single and DSBs), DNA intrastrand adducts, and DNA-protein crosslinks, all of which are cytotoxic and some can be mutagenic $(48,49)$. The results from the present study revealed that after BPA treatment, the cells 
showed an upregulation of genes involved in DNA repair, suggesting that BPA produced DNA DSBs and that the normal breast epithelial cells increased the expression of DNA repair genes to overcome the damage (Fig. 3). The normal-like human breast epithelial cell line, MCF-10F, showed an increased expression of BRCA1, BARD1, CtIP, RAD51 and BRCC3, all of which are genes involved in DNA repair, after exposure to BPA.

The human BRCA1 is a nuclear polypeptide consisting of 1,863 amino acids and it contains several functional domains that interact directly or indirectly with a variety of molecules, including tumor suppressor, oncogenes, DNA damage repair proteins, cell cycle regulators, and transcriptional activators and repressors (Fig. 3) (50). BRCA1 exists as a heterodimer with BARD1 and most of the functions of BRCA1 have been attributed to occur in association with BARD1 $(51,52)$. Disruption of the BRCA1-BARD1 interaction would impair the cell cycle checkpoint control as well as DNA repair functions of BRCA1 which could lead to tumorigenesis. BRCA1 ubiquitinates its phosphorylation-dependent partner, CtIP (RBBP8), and this reaction plays a role in the $\mathrm{G} 2 / \mathrm{M}$ checkpoint control upon DNA damage (53). RAD51, also involved in DNA-damage repair, interacts with BRCA1 (54). Our results showed that $B R C A 2$ was downregulated in the cells treated with $10^{-6} \mathrm{M}$ BPA; the BRCA2 protein, which has a function similar to that of BRCA1, also interacted with RAD51. By influencing DNA damage repair, BRCA1, BRCA2, and RAD51 play a role in maintaining the stability of the human genome. BRCC3 encodes a subunit of the BRCA1-BRCA2 containing complex (BRCC), which is an E3 ubiquitin ligase; this protein is also thought to be involved in the cellular response to progression through the G2/M checkpoint. Our results demonstrated that normal breast epithelial cells treated with BPA showed an increased expression of BRCAl, $B A R D 1, C t I P, R A D 51$ and BRCC3; all of which are involved in DNA repair. This supports the hypothesis that BPA can initiate breast cancer by generating adducts or reactive oxygen species (ROS) that can produce a variety of DNA modifications that, if not countered by DNA repair, can lead to cell transformation.

Our results suggest that the loss of BRCAl could lead to an increased sensitivity to BPA as it was shown by Jones et al (55). We isolated primary breast epithelial cells from a BRCAI carrier; these cells were treated continuously for 1 week with medium containing $10^{-5} \mathrm{M}$ or $10^{-6} \mathrm{M}$ BPA and, at the end of the treatment the ductulogenic and invasion assays were performed. These BRCAl mutant cells treated with BPA formed an increased number of spherical masses in collagen and showed increased invasion (data not shown). Our results suggest that women that carry BRCAl mutations could be more susceptible to the effects of BPA.

Human exposure to BPA is widespread and studies have shown detectable levels of BPA ranging from 0.2 to $10 \mathrm{ng} / \mathrm{ml}$ $(\sim 0.5-40 \mathrm{nM})$ in adult and fetal human serum (56). Although the doses of $10^{-5} \mathrm{M}$ and $10^{-6} \mathrm{M}$ BPA that were used in our studies were higher compared to the concentrations found in serum samples, the cells were exposed for 2 weeks in contrast to humans that are exposed to low doses for longer periods of time.

In conclusion, our results show that BPA induces the expression of genes related to DNA repair in normal human breast epithelial cells. The upregulation of BRCA1, BRCA2, RAD51, $B A R D I$ and $B R C C 3$ expression was induced after BPA expo- sure in MCF-10F cells. This suggests that in BRCA1 carriers, BPA exposure could lead to increased frequency of DNA mutations. Our results suggest that loss of BRCAl could lead to an increased sensitivity to BPA. Furthermore, this is the first study demonstrating that BPA induces DNA methylation changes in human breast epithelial cells.

\section{Acknowledgements}

This study was supported by the grants, R21 ES015894 and U01 ES/CA 12771, from the NIH.

\section{References}

1. Brotons JA, Olea-Serrano MF, Villalobos M, Pedraza V and Olea N: Xenoestrogens released from lacquer coatings in food cans. Environ Health Perspect 103: 608-612, 1995.

2. Olea N, Pulgar R, Perez P, et al: Estrogenicity of resin-based composites and sealants used in dentistry. Environ Health Perspect 104: 298-305, 1996.

3. Brede C, Fjeldal P, Skjevrak I and Herikstad H: Increased migration levels of bisphenol A from polycarbonate baby bottles after dishwashing, boiling and brushing. Food Addit Contam 20: 684-689, 2003.

4. Burridge E: Bisphenol A: product profile. Eur Chem News 14-20, 2003.

5. Howdeshell KL, Peterman PH, Judy BM, et al: Bisphenol A is released from used polycarbonate animal cages into water at room temperature. Environ Health Perspect 111: 1180-1187, 2003.

6. Fernandez MF, Arrebola JP, Taoufiki J, et al: Bisphenol-A and chlorinated derivatives in adipose tissue of women. Reprod Toxicol 24: 259-264, 2007.

7. Calafat AM, Kuklenyik Z, Reidy JA, Caudill SP, Ekong J and Needham LL: Urinary concentrations of bisphenol A and 4-nonylphenol in a human reference population. Environ Health Perspect 113: 391-395, 2005

8. Ikezuki Y, Tsutsumi O, Takai Y, Kamei Y and Taketani Y: Determination of bisphenol A concentrations in human biological fluids reveals significant early prenatal exposure. Hum Reprod 17: 2839-2841, 2002 .

9. Matsumoto A, Kunugita N, Kitagawa K, et al: Bisphenol A levels in human urine. Environ Health Perspect 111: 101-104, 2003.

10. Ouchi K and Watanabe S: Measurement of bisphenol A in human urine using liquid chromatography with multi-channel coulometric electrochemical detection. J Chromatogr B Analyt Technol Biomed Life Sci 780: 365-370, 2002.

11. Schonfelder G, Wittfoht W, Hopp H, Talsness CE, Paul M and Chahoud I: Parent bisphenol A accumulation in the human maternal-fetal-placental unit. Environ Health Perspect 110: A703-A707, 2002.

12. Yang M, Kim SY, Lee SM, et al: Biological monitoring of bisphenol A in a Korean population. Arch Environ Contam Toxicol 44: 546-551, 2003.

13. Keri RA, Ho SM, Hunt PA, Knudsen KE, Soto AM and Prins GS: An evaluation of evidence for the carcinogenic activity of bisphenol A. Reprod Toxicol 24: 240-252, 2007.

14. Soto AM, Maffini MV and Sonnenschein C: Neoplasia as development gone awry: the role of endocrine disruptors. Int J Androl 31: 288-293, 2008.

15. Jenkins S, Raghuraman N, Eltoum I, Carpenter M, Russo J and Lamartiniere CA: Oral exposure to bisphenol a increases dimethylbenzanthracene-induced mammary cancer in rats. Environ Health Perspect 117: 910-915, 2009.

16. Dairkee SH, Seok J, Champion S, et al: Bisphenol A induces a profile of tumor aggressiveness in high-risk cells from breast cancer patients. Cancer Res 68: 2076-2080, 2008.

17. Diel P, Olff S, Schmidt S and Michna H: Effects of the environmental estrogens bisphenol A, o,p'-DDT, p-tert-octylphenol and coumestrol on apoptosis induction, cell proliferation and the expression of estrogen sensitive molecular parameters in the human breast cancer cell line MCF-7. J Steroid Biochem Mol Biol 80: $61-70,2002$

18. Singleton DW, Feng Y, Yang J, Puga A, Lee AV and Khan SA: Gene expression profiling reveals novel regulation by bisphenol-A in estrogen receptor-alpha-positive human cells. Environ Res 100: 86-92, 2006. 
19. Soto AM, Sonnenschein C, Chung KL, Fernandez MF, Olea N and Serrano FO: The E-SCREEN assay as a tool to identify estrogens: an update on estrogenic environmental pollutants. Environ Health Perspect 103 (Suppl 7): 113-122, 1995.

20. Hugo ER, Brandebourg TD, Woo JG, Loftus J, Alexander JW and Ben-Jonathan N: Bisphenol A at environmentally relevant doses inhibits adiponectin release from human adipose tissue explants and adipocytes. Environ Health Perspect 116: 1642-1647, 2008.

21. Fernandez SV and Russo J: Estrogen and xenoestrogens in breast cancer. Toxicol Pathol 38: 110-122, 2010.

22. Kocdor H, Kocdor MA, Russo J, et al: Human chorionic gonadotropin (hCG) prevents the transformed phenotypes induced by 17 beta-estradiol in human breast epithelial cells. Cell Biol Int 33 : 1135-1143, 2009

23. Weber M, Davies JJ, Wittig D, et al: Chromosome-wide and promoter-specific analyses identify sites of differential DNA methylation in normal and transformed human cells. Nat Genet 37: 853-862, 2005.

24. Irizarry R, Hobbs B, Collin F, et al: Exploration, normalization, and summaries of high density oligonucleotide array probe level data. Biostatistics 4: 249-264, 2003.

25. Smyth GK: Linear models and empirical Bayes methods for assessing diferential expression in microarray experiments. Stat Appl Genet Mol Biol 3: Article 3, 2004.

26. Ji H, Jiang H, Ma W, Johnson DS, Myers RM and Wong WH: An integrated software system for analyzing ChIP-chip and ChIP-seq data. Nat Biotechnol 26: 1293-1300, 2008.

27. Johnson WE, Li W, Meyer CA, et al: Model-based analysis of tiling-arrays for ChIP-chip. Proc Natl Acad Sci USA 103: 12457-12462, 2006

28. Jaenisch R and Bird A: Epigenetic regulation of gene expression: how the genome integrates intrinsic and environmental signals Nat Genet 33 (Suppl): 245-254, 2003.

29. Ho SM, Tang WY, Belmonte de Frausto J and Prins GS Developmental exposure to estradiol and bisphenol A increases susceptibility to prostate carcinogenesis and epigenetically regulates phosphodiesterase type 4 variant 4 . Cancer Res 66 : 5624-5632, 2006.

30. Dolinoy DC, Huang D and Jirtle RL: Maternal nutrient supplementation counteracts bisphenol A-induced DNA hypomethylation in early development. Proc Natl Acad Sci USA 104: 13056-13061, 2007.

31. O'Connor L, Strasser A, O'Reilly LA, et al: Bim: a novel member of the Bcl-2 family that promotes apoptosis. EMBO J 17: 384-395, 1998.

32. Varma AK, Brown RS, Birrane G and Ladias JA: Structural basis for cell cycle checkpoint control by the BRCA1-CtIP complex. Biochemistry 44: 10941-10946, 2005.

33. Cavalieri EL and Rogan EG: Is bisphenol A a weak carcinogen like the natural estrogens and diethylstilbestrol? IUBMB Life 62 746-751, 2010.

34. Matthews JB, Twomey $\mathrm{K}$ and Zacharewski TR: In vitro and in vivo interactions of bisphenol A and its metabolite, bisphenol A glucuronide, with estrogen receptors alpha and beta. Chem Res Toxicol 14: 149-157, 2001.

35. Routledge EJ, White R, Parker MG and Sumpter JP: Differential effects of xenoestrogens on coactivator recruitment by estrogen receptor (ER) alpha and ERbeta. J Biol Chem 275: 35986-35993, 2000.

36. Maruyama S, Fujimoto N, Yin H and Ito A: Growth stimulation of a rat pituitary cell line MtT/E-2 by environmental estrogens in vitro and in vivo. Endocr J 46: 513-520, 1999.

37. Cavalieri E, Chakravarti D, Guttenplan J, et al: Catechol estrogen quinones as initiators of breast and other human cancers: implications for biomarkers of susceptibility and cancer prevention. Biochim Biophys Acta 1766: 63-78, 2006.
38. Cavalieri E, Frenkel K, Liehr JG, Rogan E and Roy D: Estrogens as endogenous genotoxic agents - DNA adducts and mutations. J Natl Cancer Inst Monogr 75-93, 2000.

39. Cavalieri E, Kohli E, Zahid M and Rogan E: Greater reactivity of estradiol-3,4-quinone vs estradiol-2,3-quinone with DNA in the formation of depurinating DNA adducts. Proc Amer Assoc Cancer Res 44: 180, 2003.

40. Liehr JG: Is estradiol a genotoxic mutagenic carcinogen? Endocr Rev 21: 40-54, 2000

41. Russo J, Fernandez SV, Russo PA, et al: 17-Beta-estradiol induces transformation and tumorigenesis in human breast epithelial cells. FASEB J 20: 1622-1634, 2006.

42. Izzotti A, Kanitz S, D'Agostini F, Camoirano A and De Flora S: Formation of adducts by bisphenol A, an endocrine disruptor, in DNA in vitro and in liver and mammary tissue of mice. Mutat Res 679: 28-32, 2009.

43. Bentley P, Bieri F, Kuster H, et al: Hydrolysis of bisphenol A diglycidylether by epoxide hydrolases in cytosolic and microsomal fractions of mouse liver and skin: inhibition by bis epoxycyclopentylether and the effects upon the covalent binding to mouse skin DNA. Carcinogenesis 10: 321-327, 1989.

44. Steiner S, Honger G and Sagelsdorff P: Molecular dosimetry of DNA adducts in $\mathrm{C} 3 \mathrm{H}$ mice treated with bisphenol A diglycidylether. Carcinogenesis 13: 969-972, 1992.

45. Vanhoutte K, Van Dongen W, Hoes I, et al: Development of a nanoscale liquid chromatography/electrospray mass spectrometry methodology for the detection and identification of DNA adducts. Anal Chem 69: 3161-3168, 1997.

46. Atkinson A and Roy D: In vitro conversion of environmental estrogenic chemical bisphenol A to DNA binding metabolite(s). Biochem Biophys Res Commun 210: 424-433, 1995.

47. Chitra KC, Latchoumycandane $\mathrm{C}$ and Mathur PP: Induction of oxidative stress by bisphenol $\mathrm{A}$ in the epididymal sperm of rats. Toxicology 185: 119-127, 2003.

48. Powell CL, Swenberg JA and Rusyn I: Expression of base excision DNA repair genes as a biomarker of oxidative DNA damage. Cancer Lett 229: 1-11, 2005.

49. Hazra TK, Das A, Das S, Choudhury S, Kow YW and Roy R Oxidative DNA damage repair in mammalian cells: a new perspective. DNA Repair (Amst) 6: 470-480, 2007.

50. Deng CX and Brodie SG: Roles of BRCA1 and its interacting proteins. Bioessays 22: 728-737, 2000.

51. Mallery DL, Vandenberg CJ and Hiom K: Activation of the E3 ligase function of the BRCA1/BARD1 complex by polyubiquitin chains. EMBO J 21: 6755-6762, 2002

52. Wu LC, Wang ZW, Tsan JT, et al: Identification of a RING protein that can interact in vivo with the BRCA1 gene product. Nat Genet 14: 430-440, 1996.

53. Yu X and Chen J: DNA damage-induced cell cycle checkpoint control requires CtIP, a phosphorylation-dependent binding partner of BRCA1 C-terminal domains. Mol Cell Biol 24: 9478-9486, 2004.

54. Chen JJ, Silver D, Cantor S, Livingston DM and Scully R: BRCA1, BRCA2, and Rad51 operate in a common DNA damage response pathway. Cancer Res 59: 1752s-1756s, 1999.

55. Jones LP, Sampson A, Kang HJ, et al: Loss of BRCA1 leads to an increased sensitivity to Bisphenol A. Toxicol Lett 199: 261-268, 2010.

56. Welshons WV, Nagel SC and vom Saal FS: Large effects from small exposures. III. Endocrine mechanisms mediating effects of bisphenol A at levels of human exposure. Endocrinology 147: S56-S69, 2006. 